Revue des patrimoines

33 | 2017

Patrimoine culturel immatériel et institutions patrimoniales

\title{
Patrimoine culturel immatériel et institutions patrimoniales \\ Éditorial
}

\section{Séverine Cachat et Christian Hottin}

\section{(2) OpenEdition Journals}

Édition électronique

URL : http://journals.openedition.org/insitu/15619

DOI : 10.4000/insitu. 15619

ISSN : $1630-7305$

Éditeur

Ministère de la Culture

\section{Référence électronique}

Séverine Cachat et Christian Hottin, «Patrimoine culturel immatériel et institutions patrimoniales », In Situ [En ligne], 33 | 2017, mis en ligne le 09 novembre 2017, consulté le 24 septembre 2020. URL : http://journals.openedition.org/insitu/15619 ; DOI : https://doi.org/10.4000/insitu.15619

Ce document a été généré automatiquement le 24 septembre 2020.

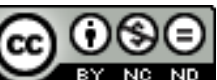

In Situ Revues des patrimoines est mis à disposition selon les termes de la licence Creative Commons Attribution - Pas d'Utilisation Commerciale - Pas de Modification 4.0 International. 


\title{
Patrimoine culturel immatériel et institutions patrimoniales
}

\author{
Éditorial
}

Séverine Cachat et Christian Hottin

\section{Le silence des textes ${ }^{1}$}

1 La thématique de ce numéro d'In Situ a trouvé, alors même que les articles étaient en cours de collecte et de relecture, une réponse législative aussi lapidaire qu'éclairante. Par l'article 55 de la loi «Liberté de création, architecture et patrimoine » (LCAP) se trouve modifié l'article L.1 du code du Patrimoine qui porte sur la définition de cette notion. Depuis l'adoption de la loi LCAP, le patrimoine «s'entend également des éléments du patrimoine culturel immatériel, au sens de l'article 2 de la convention internationale pour la Sauvegarde du patrimoine culturel immatériel adoptée à Paris le 17 octobre 2003 ». Pleinement incorporé dans la définition canonique du patrimoine, le patrimoine culturel immatériel $(\mathrm{PCI})$ demeure néanmoins à la marge extrême de l'institution patrimoniale, ou plus exactement, des institutions qui l'ont construite par agrégations successives, puisque rien n'est dit du PCI dans les sept livres qui composent le code et qui, presque tous, traitent spécifiquement de l'une ou l'autre de ces institutions : archéologie, archives, musées, Monuments historiques, bibliothèques.

2 Malgré cela, le ministère de la Culture a entrepris, dès la ratification de la convention de l'Unesco par la France, en 2006, et donc bien avant cette inclusion dans la loi, d'élaborer les modalités de gestion et de valorisation de ce nouveau champ patrimonial (outils, réseaux, processus) et de développer, avec l'ensemble des services et organismes associés à cette politique, une expertise à l'échelle nationale. Mais l'exposé des pratiques, que l'on privilégie ici, incarnera de façon plus explicite, il est vrai, la grande richesse opérative d'un secteur patrimonial si rapidement décrit par le législateur, une décennie plus tard. 
Figure 1

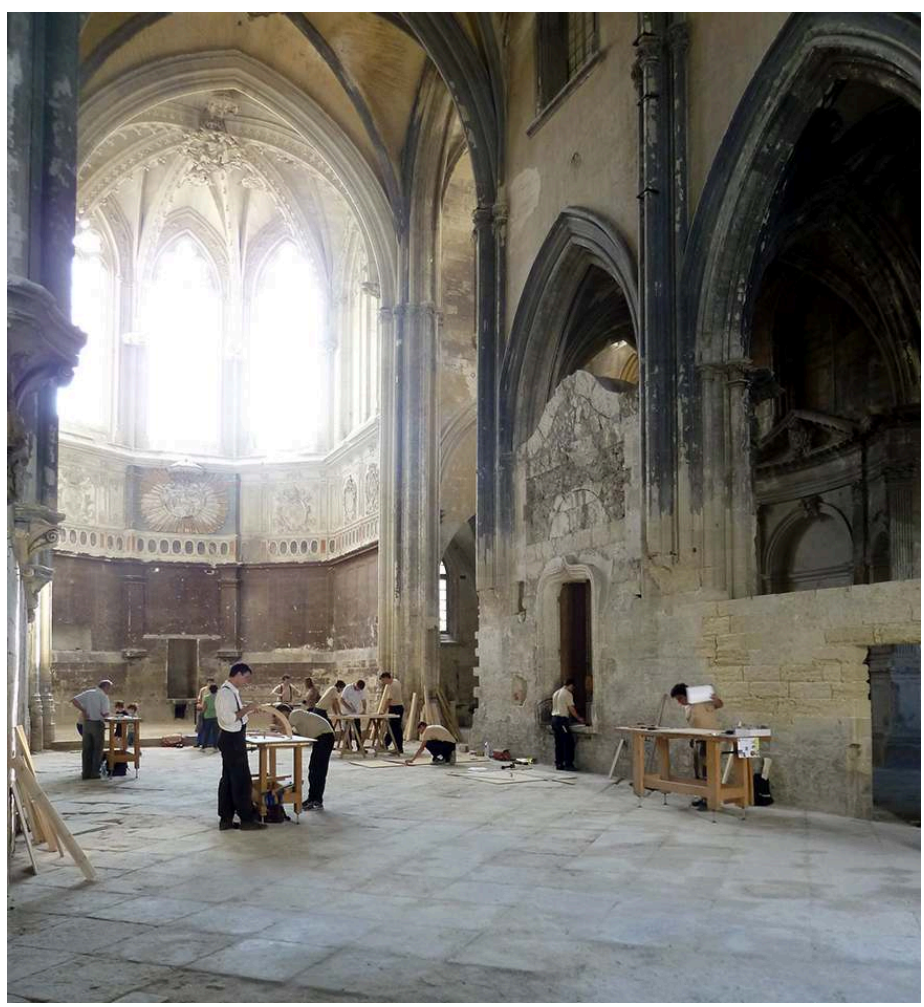

Le compagnonnage, réseau de transmission des savoirs et des identités par le métier.

(c) JPC, FNCMB (Fédération Compagnonnique - Compagnons du Tour de France), 2014.

\section{Une préoccupation transnationale}

3 La question de la réception et de l'interprétation par les États de la convention de l'Unesco pour la Sauvegarde du patrimoine culturel immatériel (PCI) est sans doute l'un des sujets les plus prisés de celles et ceux qui, depuis une quinzaine d'années, se préoccupent d'étudier ou de mettre en œuvre le texte de l'Unesco. Pour les savants de toutes les disciplines des sciences humaines et sociales, il y a là un magnifique objet d'étude ${ }^{2}$. Pour les responsables administratifs en charge de l'application des principes généraux donnés par l'Unesco, il y a là une clé pour évaluer l'originalité des actions conduites dans différents pays et apprécier la pertinence d'une transposition dans le leur ${ }^{3}$.

4 En France, ce sujet fut présent dès le milieu des années 2000, lorsque la convention fut approuvée et que débuta sa mise en œuvre par le ministère de la Culture. Tandis que la mission Ethnologie, service de la direction de l'Architecture et du Patrimoine en charge de la convention, exposait la manière dont allaient être déclinés ses articles relatifs à la mise en œuvre au plan national, une réflexion approfondie était conduite par des chercheurs en sciences sociales sur les formes de continuité ou de discontinuité existant entre le dispositif prôné par l'Unesco et celui, antérieur, du " patrimoine ethnologique ». Plus généralement, la relation entre ethnologie et patrimoine culturel immatériel fut alors explorée de diverses manières à travers des séminaires, colloques, journées d'études, mais aussi recherches appliquées ou impliquées. 
5 Pour passionnants et salutaires qu'ils soient, ces échanges n'en laissaient pas moins de côté la question de la réception et de l'appropriation - éventuelle - de la convention par les autres secteurs de l'institution patrimoniale. En quoi ce texte, avec son objet et ses orientations propres, pouvait-il avoir une influence sur les manières de faire patrimoine dans les musées, les archives ou les Monuments historiques?

\section{L'élaboration d'un questionnement}

6 Se plaçant sur le terrain des études de cas concrets et des retours d'expérience, l'Institut national du patrimoine organisa le 10 juin 2013, en partenariat avec la direction générale des Patrimoines (département du pilotage de la recherche et de la politique scientifique), une journée de formation ouverte dédiée à la question du patrimoine culturel immatériel et des métiers du patrimoine ${ }^{4}$. Très suivie, cette rencontre permit à des professionnels de l'Inventaire général, des archives, des musées ou encore de l'archéologie d'exposer des projets ou des réalisations qui, selon les cas, faisaient appel aux spécificités méthodologiques propres au PCI (la participation des communautés), ou, plus simplement, se concentraient sur l'étude de ses objets (les pratiques traditionnelles vivantes).

7 Dans la continuité de ce premier rendez-vous, la revue In Situ lança en 2014 un appel à contribution sur le thème du PCI et des institutions patrimoniales, avec, comme problématique centrale, la question des changements induits par cette nouvelle vision du patrimoine : « Dans quelle mesure la nouvelle démarche patrimoniale prônée par les instances internationales offre-t-elle l'opportunité d'un renouvellement des discours et des formes d'action patrimoniale? Comment les chercheurs, administrateurs et professionnels du patrimoine se positionnent-ils par rapport à ce tournant participatif qui, en remettant en cause le traitement expert du patrimoine, les invite à renégocier leur relation avec ces "nouveaux" acteurs et à repenser leur pratique? Quelles compétences, méthodologies et outils ces derniers peuvent-ils mobiliser ou ont-ils développés pour identifier, inventorier, documenter, exposer, valoriser le PCI ? ${ }^{56}$.

Presque simultanément, la réflexion de plusieurs acteurs s'est portée sur la relation qui pouvait être établie entre le $\mathrm{PCI}$ et l'un des moyens privilégiés de monstration du patrimoine, historiquement très légitime et rationnellement attaché à ce segment particulièrement valorisé de l'institution que sont les musées, à savoir l'exposition. En effet, dans le prolongement d'une participation de plusieurs années à l'Inventaire du PCI en France, l'université de Pau et des Pays de l'Adour a mis sur pied, en partenariat avec le musée d'Ethnologie de l'Université de Bordeaux une exposition sur le PCI en Aquitaine, "(Extra)ordinaire quotidien». La tenue de cette manifestation, en 2014, s'accompagna de l'organisation d'une journée d'études soutenue par la direction générale des Patrimoines ${ }^{7}$. Il y avait là un prolongement très naturel à la problématique plus générale de l'appel à contribution d'In Situ. 
Figure 2

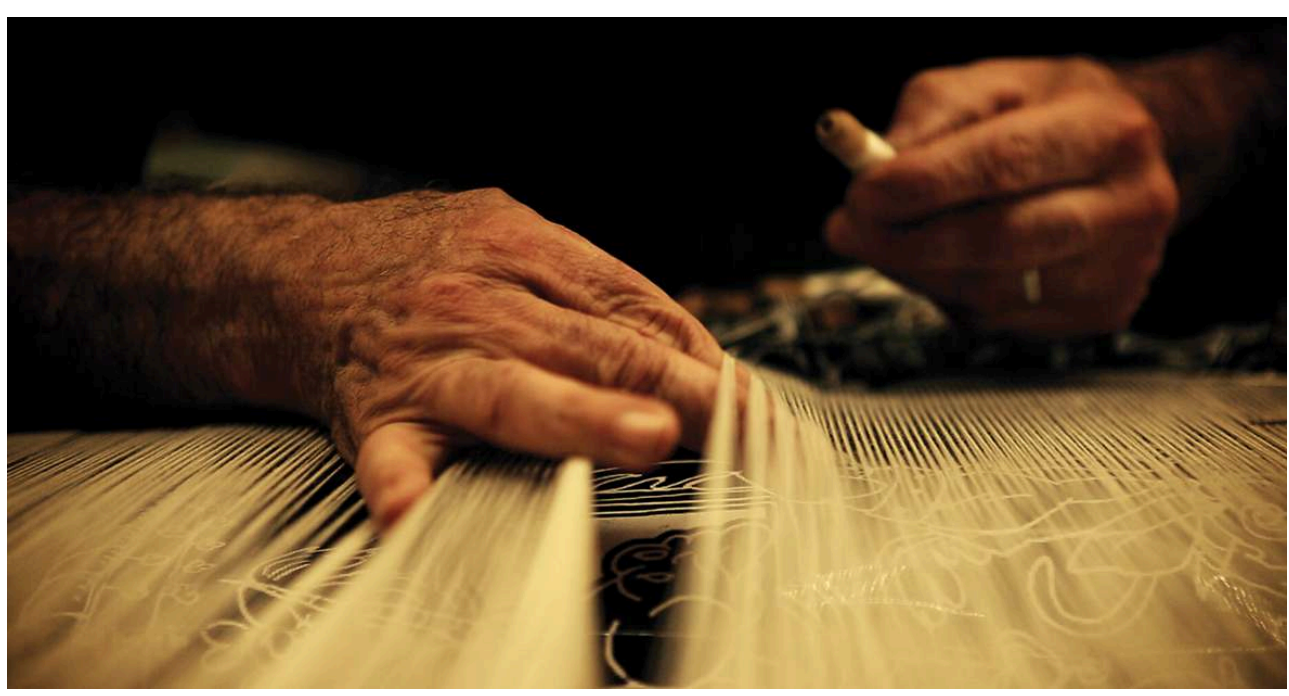

La tapisserie d'Aubusson.

(c)RE_ServiceCommunicationCreuseGrandSud_2013

\section{« Le PCI. Nouvelles expériences en France »?}

Paraissant en 2017, plus de dix ans après la ratification française, ce numéro d'In Situ peut être considéré, au moins en partie, comme une nouvelle réflexion collective sur les expériences françaises en matière de $\mathrm{PCI}$, six ans après la publication du numéro 25 de L'Internationale de l'imaginaire ${ }^{8}$. Entre les deux publications, la diversification des expériences, mais aussi celle des points de vue, est frappante. Alors que dominaient presque sans partage les analyses et les retours d'expérience liés aux candidatures ou à la mise en place de l'Inventaire du PCI en France, se manifeste désormais la diversité des modes d'appropriation de la convention et de ses outils : l'inventaire, bien entendu, reste une des composantes fondamentales de la mise en œuvre de cette politique ${ }^{9}$ mais chantiers de numérisation ${ }^{10}$, de médiation ou d'exposition ${ }^{11}$ en constituent désormais souvent le prolongement.

10 Cette diversification des modes d'appropriation de la convention va néanmoins de pair avec la persistance d'une relation privilégiée entre le patrimoine culturel immatériel et l'ethnologie. De fait, les autres disciplines ou secteurs de l'institution du patrimoine, bien que sollicités par le biais de l'appel à contribution, ont relativement peu répondu à celui-ci tandis que la mobilisation des chercheurs, professionnels de la culture et cadres associatifs issus de cette discipline ou proches d'elle répondaient à l'appel et faisaient des propositions. Ainsi, le PCI continue de se manifester pour les ethnologues français sous une double apparence : celle d'un champ d'application et d'implication possible à partir de la recherche académique et celle d'un objet de recherche dont l'ethnologue pourrait se saisir, la diversité des parcours personnels et la complexité des positionnements institutionnels autorisant toutes les synthèses et créations possibles à partir de ces deux pôles ${ }^{12}$, et notamment la poursuite et l'approfondissement de démarches d'action participatives qui, cherchant à « coller au plus près » de l'esprit de la convention, constituent sans doute l'un des apport les plus originaux du travail des ethnologues sur le $\mathrm{PCI}^{13}$. 
Figure 3

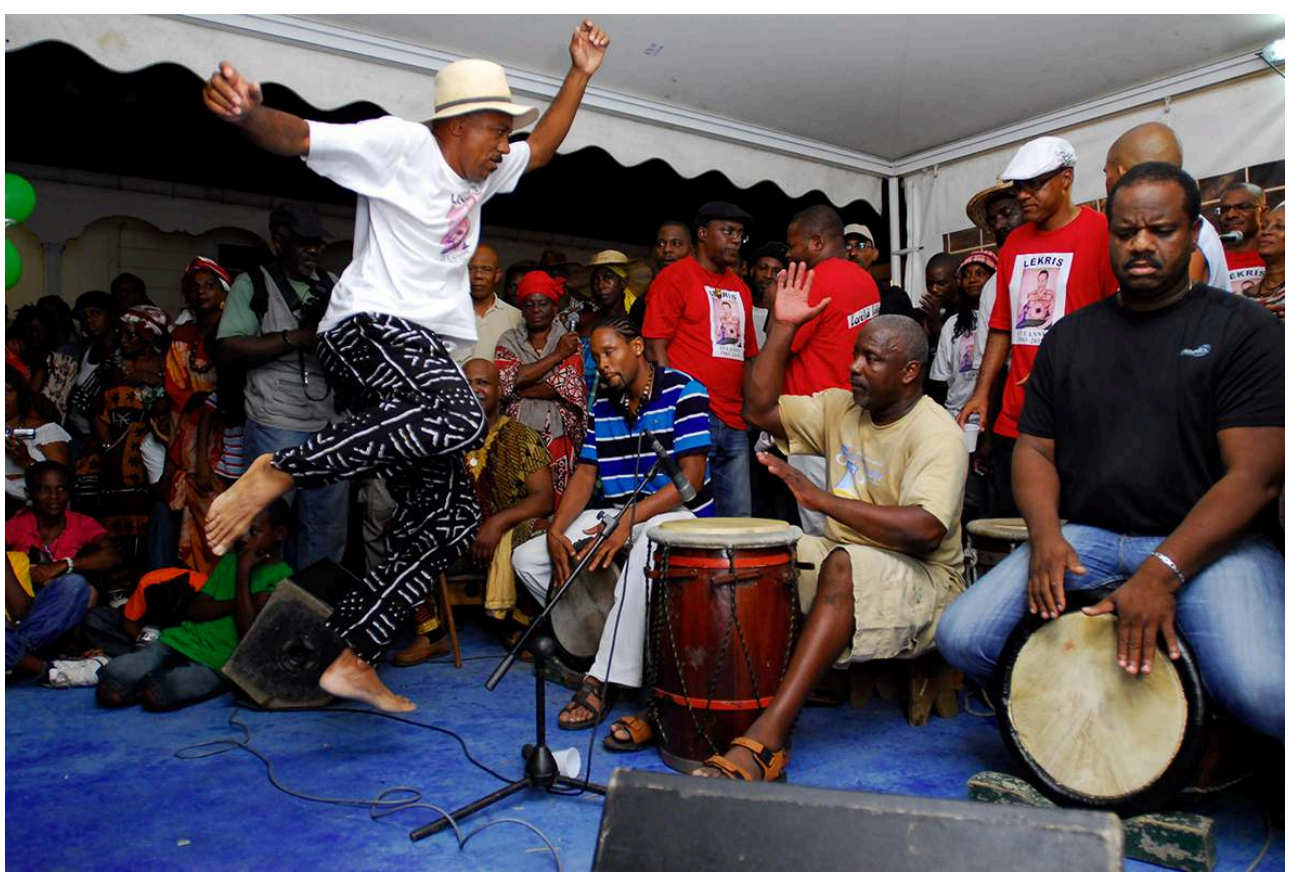

Le Gwoka. Face à face dansè-makè (danseur-tambourier).

(c) de Bompuis, 2010.

11 Ainsi, si les textes restent - presque - silencieux sur la question du PCI, ce numéro d' In Situ montrera à ses lecteurs que le patrimoine immatériel est désormais bien présent au sein des dispositifs et des pratiques de l'institution patrimoniale. Il est le lieu d'un dialogue renouvelé entre les ethnologues et celle-ci, une nouvelle manière de s'impliquer sans craindre parfois de se couler dans des dispositifs « unesquiens » qui avaient de prime abord été jugés peu compatibles avec les exigences scientifiques de la discipline. Il est aussi l'occasion, pour les ethnologues, de s'investir résolument dans des pratiques typiques de l'institution: expositions, inventaire, projets de numérisation. Si au commencement de l'aventure, en 2006, un ethnologue du ministère a pu avancer, non sans perspicacité, que « le patrimoine immatériel, c'est le patrimoine ethnologique sans les ethnologues », il semblerait que cette réserve ne soit plus de mise désormais et qu'elle ait cédé la place à une grande diversité de modes d'appropriation où chacun, dans le respect des postures de l'autre, trouve à servir la cause.

\section{NOTES}

1. - Nous souhaitons remercier vivement, à l'occasion de la parution de ce numéro d'In Situ, Françoise Cosler, pour son travail et son écoute tout au long de la préparation du dossier, ainsi que Catherine Gros, pour ses relectures très attentives et ses propositions toujours judicieuses. 
2. - Voir à ce propos, par exemple, le programme de recherche OSMOSE, conduit par l'Académie de la culture de Lettonie et l'Institut des sciences sociales du politique (ENS-Cachan). L'objectif de cette recherche est de construire une méthodologie de droit comparé adaptée au patrimoine culturel immatériel. Voir sur le site: http://dpc.hypotheses.org/le-projet-osmose [consulté le 06/11/2017].

3. - C'est l'objet du colloque annuel organisé par l'ethnopôle de Vitré, en particulier de la première session (2012) sur "L'administration du PCI en Europe ", dont les actes ont été édités dans la revue en ligne des Cahiers du CFPCI, voir : www.cfpci.fr/actualites/cahier-du-cfpci-1 [consulté le 06/11/2017].

4. - Le programme est consultable en ligne, ainsi qu'un enregistrement audio des débats : http:// www.culturecommunication.gouv.fr/Thematiques/Patrimoine-ethnologique/Soutien-a-larecherche/Colloques-seminaires-et-journees-d-etude/2013/Journee-d-etude-et-de-formationPatrimoine-culturel-immateriel-et-metiers-du-patrimoine-a-l-INP.

5. - Voir le texte complet de l'appel à contribution: http://www.cfpci.fr/actualites/appel-acontribution-in-situ [consulté le 06/11/2017].

6. - Ce questionnement est à l'origine du projet Europe créative 2017-2020 «Intangible cultural heritage \& museums project» porté par cinq ONG en Europe: Tapis-plein (Belgique), Kenniscentrum Immaterieel Erfgoed Nederland (Pays-Bas), Simbdea (Italie), la Maison des Cultures du Monde-CFPCI (France) ainsi que l'Association des musées suisses avec l'Office fédéral de la Culture (Suisse). Ce projet vise à produire et diffuser un certain nombre d'outils communs favorisant la prise en compte du PCI dans les musées et les approches participatives. Voir le site : www.ichandmuseums.eu/en [consulté le 07/11/2017].

7. - Voir le programme sur le site du laboratoire ITEM: http://item.hypotheses.org/tag/ exposition?lang=en_GB [consulté le 06/11/2017].

8. - Le Patrimoine culturel immatériel. Premières expériences en France. Arles/Paris : Actes Sud/Maison des cultures du monde, coll. « Internationale de l'imaginaire », 2011.

9. - Voir dans ce numéro la contribution de Laurent Sébastien Fournier, "L'expérience de l'inventaire, une opportunité pour la recherche anthropologique », In Situ [En ligne], 33 |2017, mis en ligne le 30 octobre 2017, consulté le 06 novembre 2017. URL : http://insitu.revues.org/ 15465.

10. - Voir dans ce numéro la contribution de Jean-Jacques Castéret, «Le numérique comme « lieu » de la sauvegarde du Patrimoine culturel immatériel », In Situ [En ligne], 33 | 2017, mis en ligne le 02 novembre 2017, consulté le 06 novembre 2017. URL : http://insitu.revues.org/15478.

11. - Voir notamment dans ce numéro les contributions de Lucia Bienvenu et Patricia HeinigerCasteret, « « (Extra)ordinaire quotidien » : exposer le patrimoine culturel immatériel », In Situ [En ligne], $33 \mid 2017$, mis en ligne le 09 novembre 2017, consulté le 09 novembre 2017. URL: http://insitu.revues.org/15648; Valérie Perlès, « «Sortez des clichés! Regard sur des patrimoines vivants » », In Situ [En ligne], 33 | 2017, mis en ligne le 06 novembre 2017, consulté le 06 novembre 2017. URL : http://insitu.revues.org/15616; Noël Barbe et Jean-Christophe Sevin, "Curateur ou traducteur? Exposer le patrimoine culturel immatériel en Alsace Bossue », In Situ [En ligne], 33 |2017, mis en ligne le 03 novembre 2017, consulté le 06 novembre 2017. URL: http://insitu.revues.org/15596 et Charles Quimbert, «Exposer le PCI : l'exemple de l'exposition itinérante « À la découverte du PCI en Bretagne » ", In Situ [En ligne], 33 | 2017, mis en ligne le 03 novembre 2017, consulté le 06 novembre 2017. URL : http://insitu.revues.org/15529.

12. - Voir, à ce propos les contributions dans ce numéro de Noël Barbe autour d'une exposition sur le PCI en Alsace Bossue et sur une candidature à l'Unesco pour le «Biou " d'Arbois (étude rédigée avec Flavie Ailhaud) : Flavie Ailhaud et Noël Barbe, « S'attacher/être attaché au biou », In Situ [En ligne], 33 | 2017, mis en ligne le 02 novembre 2017, consulté le 06 novembre 2017. URL : http://insitu.revues.org/15514. 
13. - Voir à ce propos, outre les contributions citées ci-dessus, celles de Caroline Darroux, «La Maison du patrimoine oral de Bourgogne : actualité de l'activité patrimoniale et action citoyenne en région », In Situ [En ligne], 33|2017, mis en ligne le 02 novembre 2017, consulté le 06 novembre 2017. URL: http://insitu.revues.org/15496 et Jean-Louis Tornatore, «Patrimoine vivant et contributions citoyennes. Penser le patrimoine « devant » l'Anthropocène », In Situ [En ligne], 33 | 2017, mis en ligne le 03 novembre 2017, consulté le 06 novembre 2017. URL : http:// insitu.revues.org/15606.

\section{AUTEURS}

\section{SÉVERINE CACHAT}

Directrice de la Maison des cultures du Monde et du Centre français du PCI (ethnopôle)

direction@maisondesculturesdumonde.org

\section{CHRISTIAN HOTTIN}

Conservateur en chef du patrimoine, directeur des études du département des conservateurs, institut national du Patrimoine, membre de l'IIAC (LAHIC) christian.hottin@inp.fr 